\section{Facharztprüfung zur Erlangung des Facharzttitels pädiatrische Nephrologie}

Aufgrund des Weiterbildungsprogrammes, welches am 1. Januar 1998 in Kraft gesetzt wurde, ist die Teilnahme an der Facharztprüfung für diejenigen Kandidatinnen und Kandidaten Voraussetzung für die Erlangung des Facharzttitels FMH für pädiatrische Nephrologie, welche ihre Weiterbildung in pädiatrischer Nephrologie bis am 31. Dezember 1998 nicht abgeschlossen hatten. Es empfiehlt sich, die Facharztprüfung frühestens im letzten Jahr der reglementarischen Weiterbildung abzulegen (Art. 22 WBO).
Ort und Zeitpunkt: Werden nach Eingang der Anmeldungen, abhängig von gewünschter Prüfungssprache, festgelegt.

Prüfungsgebühr: Die Fachgesellschaft erhebt einen Unkostenbeitrag von Fr. 300.-.

Anmeldung:

Prof. E. Girardin,

Unité de Néphrologie-métabolisme pédiatrique, HUG, rue Willy-Donzé 6, 1211 Genève 14, Tel. 02238246 34, Fax 0223824505.

Anmeldefrist: 30. August 2000

\section{Examen de spécialiste en vue de l'obtention du titre de spécialiste FMH en néphrologie pédiatrique}

Conformément au programme de formation postgraduée entré en vigueur le $1^{\text {er }}$ janvier 1998, la participation à l'examen de spécialiste est une condition requise pour les candidats à l'obtention du titre de spécialiste FMH en néphrologie pédiatrique qui n'avaient pas terminé leur formation postgraduée en néphrologie pédiatrique le 31 décembre 1998. Il leur est recommandé de passer l'examen de spécialiste au plus tôt durant la dernière année de leur formation postgraduée réglementaire (art. 22 RFP).
Lieu et date: Ils seront fixés après réception des inscriptions et dépendront de la langue d'examen souhaitée.

Taxe d'examen: La société de discipline médicale prélève une participation aux frais de Fr. 300.-.

\section{Inscription:}

Auprès du Prof. E. Girardin,

Unité de Néphrologie-métabolisme pédiatrique, HUG, rue Willy-Donzé 6, 1211 Genève 14, tél. 02238246 34, fax 0223824505 .

Délai d'inscription: le 30 août 2000 